\title{
Coupling Vibration Model of Submerged Floating Tunnel in Space
}

\author{
Li Changzhao ${ }^{1,2} \cdot$ Zhang Mengying $^{1,2} \cdot$ Liu Liquan $^{1,2} \cdot$ Cheng Yu $^{1,2} \cdot$ Han Fei $^{3}$. \\ Zang Jubao ${ }^{4}$
}

Received: 27 October 2021 / Accepted: 12 January 2022 / Published online: 21 February 2022

(c) The Author(s) 2022

\begin{abstract}
Submerged floating tunnel is a new type of traffic building structure under water surface which can span various water courses. The structure has the characteristics of high flexibility and low damping so that vibration problems would be caused by hydrodynamic excitation. Long-term vibration problems could affect the safety and durability of the structure. Therefore, it is necessary to accurately understand the dynamic behavior of the floating tunnel under hydrodynamic excitation. This paper proposes a simplified three-dimensional model to study vibration of the structure for the excitation. Based on the Galerkin method and the mode superposition method with the boundary conditions, the dynamic differential equations of the structure were established in Generalized coordinate system. The Newmark $-\beta$ method is used to solve the equations. Then, adopting FEA (Finite Element Analysis) method to establish the finite element model of the submerged floating tunnel. The result verified the accuracy of the present model. Based on the present model for parameter analysis, the following conclusions are: displacement coordination at the connection position affects the cables' dynamic behavior greatly compared to tube. Current and horizontal component of wave have significant impact on dynamic response of tube. While the vertical wave component influences cable greatly.
\end{abstract}

Keywords Submerged floating tunnel $\cdot$ Coupled vibration - Dynamic model $\cdot$ Wave and current

Han Fei

hanfei@nwpu.edu.cn

1 JSTI Group Co., Ltd., Nanjing 211112, Jiangsu, China

2 State Key Laboratory of Safety and Health for In-Service Long Span Bridges, Nanjing 211112, Jiangsu, China

3 School of Mechanics, Civil Engineering and Architecture, Northwestern Polytechnical University, Xi' an 710129, Shanxi, China

4 Shanxi Provincial Land Engineering Construction Group Co., Ltd, Xi'an 710075, China 


\section{Abbreviations}

FEA Finite element analysis

MSDT Mid-span displacement of the tunnel

MSDC Mid-span displacement of the cable

CMS Component mode synthesis

\section{Introduction}

Submerged floating tunnel is a new type of traffic structure which can span various water courses and be constructed in deep ocean environment. The structure mainly includes the tube, the cables and the anchor mounds. The tube is suspended in the water by its buoyancy, gravity and the supporting force of the cables. The cables need to be connected with the anchor mounds at the bottom of the sea to realize the stability of the whole system $[1,2]$. It can be found that the suspended tunnel is less restricted by the span and water depth from structural features [3,4]. In the past several years, great achievements have been made in the construction of bridges and tunnels in China. Currently, the bridge and tunnel projects for planning and feasible studies are located in the three major channel (Taiwan Channel, Bohai Channel, Qiongzhou Channel) of China. One of the great challenges is the great depth of water. Therefore, submerged floating tunnel has bright prospect with some advantages [5]. Besides, it is also an environment-friendly project, so it has a broad prospect in the future. Since the beginning of this century, many researches on submerged floating tunnels have been carried out at home and abroad [6-9], but so far there is no complete and maturely analytical theories and methods, let alone the construction of submerged floating tunnels. One important factor is the fast changing of marine environment. what's more, the reality is more complex than had been thought $[10,11]$. Understanding the static and dynamic characteristics of the submerged floating tunnel under the action of the marine environment has great significance to its safety, applicability and serviceability.

Currently, response coupling between structures is one of the focuses of researches in various fields, For example, Zhang et al. [12] investigated on dynamic behaviors of rotor system with losseness and nonlinear supporting. Similarly, the response coupling between the pipe and the cables is a complicated problem. Relevant scholars have done a lot of related research on the different response coupling processing methods.

According to the methods of modeling cable, the numerical simulation of submerged floating tunnels can be divided into three categories: (1) cable is equivalent to spring, only considering their restoring force to the tube and ignoring the inertial force of the cable and the hydrodynamic force. Lu et al. [13] considered the influence of the slack of the cable and the sudden load caused by the cable slack- tension on the tube. In the research, the tunnel was simulated as a rigid body and the sudden change of cable force state was equivalent to the sudden change of cable stiffness. Lin et al. [14] simulated a floating tunnel as an elastic support beam and studied the tunnel's response under the coupling action of water flow and vehicle load. The paper compared the analytical results of beam modeling with the mode 
superposition method and the FEA method found that at least six orders are required to satisfy the requirement of accuracy. Xiang and Yang [15], Xiang et al. [16] proposed similar simplified models to study the response of tunnel under impact loads and cable breaks. Meanwhile, the paper compared with finite element simulations. The results showed that the simplified model has certain accuracy. (2) The interaction between cable and tube are often considered by an approximate coupling method that simulate the cable as a tension string. In cable vibration analysis, the dynamic strain can be estimated according to its cross vibration amplitude and the displacement of position of the tunnel at the upper end of the cable along the direction of the string line to obtain the dynamic cable force. Xiang and Chao [17] adopted this model to study the vortex-induced vibration of cable under the action of current, which analyzed the influence of water flow velocity, cable inclination angle and tunnel buoyancy ratio on the structure response. Chen et al. [18] used this model to study the coupled vibration analysis of submerged floating tunnel in wave and current. Besides, the paper analyzed the influence of water flow velocity, cable inclination and wave period. (3) The establishment of finite element model is a common method for dynamic and static analysis of structures. Lu and Jin [19] established a dynamic model based on a dual-rotor experiment rig. The coupling effect between the cable and tunnel is fully considered. The force balance condition and displacement coordination between the upper end of the cable and the corresponding point of tube are considered at the same time. The existing researches mainly adopt the FEA method to establish a complete model of the cable and tube. Di Pilato et al. [20] proposed a three-dimensional element model of cables for coupling analysis. Then, the three- dimensional beam elements were used to simulate the tube and conduct a coupled analysis of the suspension tunnel under the action of earthquake and wave, which focused on multi-point ground motion input and the role of extreme waves. Long et al. [21] adopted the finite element model by taking the Qiandao Lake suspension bridge as the prototype and used the fifth-order Stokes wave to consider the hydrodynamic effects to study the effects of tube length, buoyancyto-weight ratio, cable stiffness coefficient and buoyancy on the dynamic response. Martinelli et al. [22] proposed a three-dimensional modeling and analytical method for submerged floating tunnel, in which the tube adopted three-dimensional beam elements and the cables adopted 3-node isoparametric element. The Qiandao Lake Bridge was used as a prototype to study seismic space effect of inconsistent input on structural response. Cristian et al. [23] adopted the FEA method and concentrated mass method to establish an cable system model and analyzed the influence of wave slope, buoyancy-to- weight ratio, water depth and different cable arrangements on the response of the coupled system. Then, the paper compared them with the experimental results of the model. In the case of long wave period and huge wave height, there is a big deviation between numerical simulation and experimental results. Ji et al. [24] adopted this type of model to analyze a 900-m-long floating tunnel and studied different tunnel bending stiffness, cable arrangement interval, cable material and structural response under different wave conditions.

Regarding the structural coupling response of submerged floating tunnel, existing studies have also conducted a large number of model experiments, including experiments on tube, cable sections and coupled experiments on tube segment-cable 
systems. Chen et al. [25] conducted underwater vibration table experiments to study the response of submerged floating tunnel model under seismic excitation,which found that vertical earthquakes could cause greater structural response than horizontal earthquakes. The inclination of the cable has a significant impact on the system response. Chao [26] adopted computational fluid dynamics method and experiment to analyze the vortex-induced vibration characteristics of the circular cross-section of the cable. Seo et al. [27] conducted experimental observations based on a 1/148 scale elliptical tunnel model and found that the measured wave load was consistent with the numerical simulation load by using Morison's formula and the cable system has a significant impact on the tunnel's response under waves.

Through above literature review, it can be found that a large number of studies have been carried out on the dynamic behavior of submerged floating tunnel, cable and tunnel-cable coupling structure system under environmental effects in recent years, which obtains great achievement. Existing studies generally establish an overall model of tunnel-cable system by adopting FEA method. The low efficiency of the analysis method is not suitable for large-scale practical engineering analysis. Although the current simplified model calculation can obtain a certain accuracy result, there are still some differences with the actual situation. For example, most of the existing simplified models are two-dimensional models, but the structural system has a relatively huge gravitational stiffness in the vertical direction, while the cross stiffness is relatively small. Meanwhile, the structure is subjected to action of water flow in the cross direction. Therefore, the submerged floating tunnel is prone to produce displacement in the cross direction. The coupling characteristics of the structure have not been fully considered. For example, only the balance condition between the upper end of the cable and the corresponding connection of tunnel is considered, while the displacement coordination conditions are ignored. Above all, it is of great significance to study the simplified modeling of 3D tunnel-cable system with higher accuracy under this background.

When analyzing the cables, this paper adopts the mode expansion method with overlapping the shape function to solve the problem of the displacement coordination between the upper end of the cables and the pipe, so that the structure meets the conditions of dynamic balance and displacement coordination during the vibration process, and the calculation accuracy is improved. When analyzing the pipe body, a simplified model that can consider the lateral effects is established to analyze the effects of the lateral components of water flow and waves.

The remainder of this paper is organized as follows. Section 2 presents an analytical model of submerged floating tunnel with CMS method. Section 3 selects reasonable parameters and compares the calculation results of the present model and the finite element model for verification. Section 4 studies the response of the structural system under current and wave based on the present model. Section 5 concludes the paper.

\section{Establishment of Analytical Model}

Figure 1 shows a simplified model of submerged floating tunnel. In the model, the tunnel is simplified into a simply supported beam with a certain flexural rigidity, ignoring its axial deformation and torsion deformation. Every cable is equivalent 
to a linear tension string with constant tensile stiffness, which ignores its material nonlinearity and axial vibration. The influence of gravity and buoyancy on the entire structure is not considered.

As is shown in Fig. 1b, a local coordinate system $x_{i} y_{i} z_{i}$ is established for each cable. $v_{i}$ is the displacement of the No. $i$ cable along the $y_{i}$ direction. $l$ is the length of the cable. $\theta$ represents the inclination of the cable. Establishing a coordinate system $x_{t} y_{t} z_{t}$ with the left end of the tunnel as the origin to describe the position of the tunnel. The simply supported beam can vibrate along the $y_{t}$ (cross) direction and $z_{t}$ (vertical) direction. $x_{t}, y_{t}$ and $z_{t}$ are the axial, cross and vertical direction of the tunnel. $v_{t}, w_{t}$ indicate the displacement of the tunnel along the cross and vertical directions respectively. $D$ is the diameter of the tube.

The three-dimensional dynamic equations of the tunnel-cable system can be obtained by Hamilton principle [28].

$$
\begin{gathered}
m_{c} \frac{\partial^{2} v_{i}}{\partial t^{2}}+c \frac{\partial v_{i}}{\partial t}-\left(T_{i}+\Delta T_{i}\right) \frac{\partial^{2} v_{i}}{\partial x_{t}^{2}}=f_{v i}, \quad i=1,2,3 \ldots \\
E_{t} I_{t} \frac{\partial^{4} v_{t}}{\partial x_{t}^{4}}+m_{t} \frac{\partial^{2} v_{t}}{\partial t^{2}}+c_{t} \frac{\partial v_{t}}{\partial t}+2 \sum_{i=1}^{n}\left(T_{i}+\Delta T_{i}\right) \cos \theta \delta\left(x_{t}-x_{t i}\right)=f_{t y} \\
E_{t} I_{t} \frac{\partial^{4} w_{t}}{\partial x_{t}^{4}}+m_{t} \frac{\partial^{2} w_{t}}{\partial t^{2}}+c_{t} \frac{\partial w_{t}}{\partial t}+2 \sum_{i=1}^{n}\left(T_{i}+\Delta T_{i}\right) \sin \theta \delta\left(x_{t}-x_{t i}\right)=f_{t z}
\end{gathered}
$$

where $m_{c}$ is the mass per unit length of the No.i cable.c is the damping of the cable. $T_{i}$ , $\Delta T_{i}$ are the No.i cable initial force and dynamic force respectively. $f_{v i}$ is the environmental force on the No. $i$ cable. $m_{t}$ is the mass of the tunnel per unit length. $E_{t} I_{t}$ is the flexural rigidity of the tunnel. $c_{t}$ is the damping of the tunnel. $f_{t y}$ and $f_{t z}$ are the cross and vertical environmental forces of the tunnel respectively.

The mainly environmental effects of submerged floating tunnel under water are current and wave. The structure considers the effects of horizontal water flow and component of wave. The effect of wave force decays quickly with the influence of water depth. while depth of cables is relatively large. For cable, this paper only considers the effect of current with ignoring wave effect [29]. Existing studies have

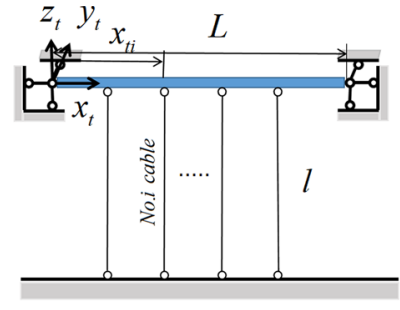

(a)

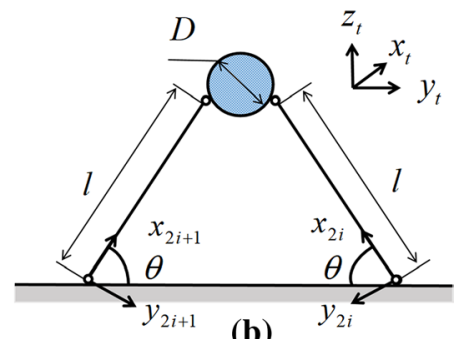

(b)

Fig. 1 Simplified model of submerged floating tunnel: a front view; b cross section 
shown that for slender structures, the effect of fluid on the structure can be calculated by the Morison formula. The Morison formula [30] indicates that the external force on the structure can be divided into the additional mass force and drag force of the fluid, which are related to relative speed and acceleration between the structure and the fluid. In this paper, Morison formula was adopted to calculate the effects of environmental load on the structural system.

$$
\begin{gathered}
f_{v, i}=-C_{m} \frac{\pi d^{2} \rho_{w}}{4} \ddot{v}_{r, i}-C_{d} \frac{d \rho_{w}}{2} \dot{v}_{r, i}\left|\dot{v}_{r, i}\right| \\
f_{t y}=-C_{m} \frac{\pi D^{2} \rho_{w}}{4} \ddot{v}_{r, t}-C_{d} \frac{D \rho_{w}}{2} \dot{v}_{r, t}\left|\dot{v}_{r, t}\right| \\
f_{t z}=-C_{m} \frac{\pi D^{2} \rho_{w}}{4} \ddot{w}_{r, t}-C_{d} \frac{D \rho_{w}}{2} \dot{w}_{r, t}\left|\dot{w}_{r, t}\right|
\end{gathered}
$$

where $C_{m}$ and $C_{d}$ are the mass additional coefficient and the viscosity coefficient respectively. Let $C_{m}=1, C_{d}=0.7$ in this paper. $\dot{v}_{r, i}, \ddot{v}_{r, i}$ represent relative velocity and relative acceleration between No.i cable and the fluid. $\dot{v}_{r, t}, \ddot{v}_{r, t}, \dot{w}_{r, t}, \ddot{w}_{r, t}$ are the relative velocity and relative acceleration between the tunnel and the fluid along the cross and vertical direction.

Airy wave theory [27] was used to study the effect of waves on the structure. Airy waves are the simplest waves. The water surface presents simple harmonic waves. The water particle makes simple harmonic motion with a fixed circular frequency. At the same time, the waves move at a certain speed. According to the Airy wave theory, the velocity field and acceleration field can be obtained as Eqs. (3a)-(3d).

$$
\begin{gathered}
u_{y}=\frac{\pi H}{T} \frac{\cosh \left[k\left(z_{0}+d\right)\right]}{\sinh (k d)} \cos \left(\omega_{w} t\right) \\
a_{y}=-\frac{\pi H \omega_{w}}{T} \frac{\cosh \left[k\left(z_{0}+d\right)\right]}{\sinh (k d)} \sin \left(\omega_{w} t\right) \\
u_{z}=\frac{\pi H}{T} \frac{\sinh \left[k\left(z_{0}+d\right)\right]}{\sinh (k d)} \sin \left(\omega_{w} t\right) \\
a_{z}=\frac{\pi H \omega_{w}}{T} \frac{\sinh \left[k\left(z_{0}+d\right)\right]}{\sinh (k d)} \cos \left(\omega_{w} t\right)
\end{gathered}
$$

where $H$ is wave height. $T_{w}$ and $d_{w}$ represent wave period and water depth respective. $K$ is wave number. $z_{0}$ and $\omega_{w}$ represent water depth and circle frequency. The tunnel is installed at a depth $z_{0}$. Defining the left cable of the cross section in Fig. 1b as odd-numbered cable, and the right cable as even-numbered cable. The displacement of the entire structural system can be approximately expressed as Eqs. (4a)-(4d) by 
adopting the mode superposition method with considering boundary coordination $[16,31]$.

$$
\begin{gathered}
v_{2 i+1}=\mathbf{N}_{c} \mathbf{V}_{2 i+1}+\frac{x_{2 i+1}}{l}\left(v_{t, 2 i+1} \sin \theta-w_{t, 2 i+1} \cos \theta\right) \\
v_{2 i}=\mathbf{N}_{c} \mathbf{V}_{2 i}-\frac{x_{2 i}}{l}\left(v_{t, 2 i} \sin \theta+w_{t, 2 i} \cos \theta\right) \\
w_{t}=\mathbf{N}_{t} \mathbf{W}_{t} \\
v_{t}=\mathbf{N}_{t} \mathbf{V}_{t}
\end{gathered}
$$

where $\mathbf{N}_{c}=\left[\sin \frac{\pi x_{i}}{l} \sin \frac{2 \pi x_{i}}{l} \cdots\right], \mathbf{N}_{t}=\left[\sin \frac{\pi x_{t}}{L} \sin \frac{2 \pi x_{t}}{L} \cdots\right] . V_{2 i+1}$ and $V_{2 i}$ are the mode coordinates of the cables respectively. $V_{t}$ and $W_{t}$ represent mode coordinates for the tunnel respectively.

Incorporating Eqs. (4a)-(4d) and into Eqs. (1a)-(1c) can get Eqs. (5a)-(5d).

$$
\begin{aligned}
& m_{c}\left(\begin{array}{l}
\mathbf{N}_{c} \ddot{\mathbf{V}}_{2 i+1}-\frac{x_{2 i+1}}{l} \ddot{w}_{t, 2 i+1} \cos \theta \\
+\frac{x_{2 i+1}}{l} \ddot{v}_{t, 2 i+1} \sin \theta
\end{array}\right)+c\left(\begin{array}{l}
\mathbf{N}_{c} \dot{\mathbf{V}}_{2 i+1}-\frac{x_{2 i+1}}{l} \dot{w}_{t, 2 i+1} \cos \theta \\
+\frac{x_{2 i+1}}{l} \dot{v}_{t, 2 i+1} \sin \theta
\end{array}\right)-\left(T_{2 i+1}+\Delta T_{2 i+1}\right) \mathbf{N}^{\prime \prime}{ }_{c} \mathbf{V}_{2 i+1}=f_{v, 2 i+1} \\
& m_{c}\left(\begin{array}{l}
\mathbf{N}_{c} \ddot{\mathbf{V}}_{2 i}-\frac{x_{2 i}}{l} \ddot{w}_{t, 2 i} \cos \theta \\
-\frac{x_{2 i}}{l} v_{t, 2 i} \sin \theta
\end{array}\right)+c\left(\begin{array}{l}
\mathbf{N}_{c} \dot{\mathbf{V}}_{2 i}-\frac{x_{2 i}}{l} \dot{w}_{t, 2 i} \cos \theta \\
-\frac{x_{2 i}}{l} \dot{v}_{t, 2 t} \sin \theta
\end{array}\right)-\left(T_{2 i}+\Delta T_{2 i}\right) \mathbf{N}_{c}^{\prime \prime} \mathbf{V}_{2 i} \\
& =f_{v, 2 i} \\
& E_{t} I_{t} \mathbf{N}_{t}^{(4)} \mathbf{V}_{t}+m_{t} \mathbf{N}_{t} \ddot{\mathbf{V}}_{t}+c_{t} \mathbf{N}_{t} \dot{\mathbf{V}}_{t}+2 \sum_{i=1}^{n}\left(T_{i}+\Delta T_{i}\right) \cos \theta \delta\left(x_{t}-x_{t i}\right)=f_{t y} \\
& E_{t} I_{t} \mathbf{N}_{t}^{(4)} \mathbf{W}_{t}+m_{t} \mathbf{N}_{t} \ddot{\mathbf{W}}_{t}+c_{t} \mathbf{N}_{t} \dot{\mathbf{W}}_{t}+2 \sum_{i=1}^{n}\left(T_{i}+\Delta T_{i}\right) \sin \theta \delta\left(x_{t}-x_{t i}\right)=f_{t z}
\end{aligned}
$$

Considering the cross amplitude of the cable and the dynamic displacement of the upper connection point of the cable along the string's direction of the cable. The dynamic cable force can be expressed as Eqs. (6a), (6b).

$$
\begin{aligned}
& \Delta T_{2 i+1}=\frac{E_{c} A_{c}}{l} \Delta l_{2 i+1}=\frac{E_{c} A_{c}}{l}\left[w_{t, 2 i+1} \sin \theta+v_{t, 2 i+1} \cos \theta+\int_{0}^{1} \frac{1}{2}\left(\frac{\partial v_{2 i+1}}{\partial x_{2 i+1}}\right)^{2} \mathrm{~d} x_{2 i+1}\right] \\
& =\frac{E_{c} A_{c}}{l}\left[w_{t, 2 i+1} \sin \theta+v_{t, 2 i+1} \cos \theta+\int_{0}^{l} \frac{1}{2}\left(\mathbf{N}_{c}^{\prime} \mathbf{V}_{2 i+1}\right)^{2} \mathrm{~d} x_{2 i+1}\right]
\end{aligned}
$$




$$
\begin{gathered}
\Delta T_{2 i}=\frac{E_{c} A_{c}}{l} \Delta l_{2 i}=\frac{E_{c} A_{c}}{l}\left[w_{t, 2 i} \sin \theta-v_{t, 2 i} \cos \theta+\int_{0}^{l} \frac{1}{2}\left(\frac{\partial v_{2 i}}{\partial x_{2 i}}\right)^{2} \mathrm{~d} x_{2 i}\right] \\
=\frac{E_{c} A_{c}}{l}\left[w_{t, 2 i} \sin \theta-v_{t, 2 i} \cos \theta+\int_{0}^{l} \frac{1}{2}\left(\mathbf{N}_{c}^{\prime} \mathbf{V}_{2 i}\right)^{2} \mathrm{~d} x_{2 i}\right]
\end{gathered}
$$

Adopting Galerkin method to simplify Eqs. (5a)-(5d) by using $\mathbf{N}_{c}$ and $\mathbf{N}_{t}$ as weighting functions for the cable and the tunnel respectively. Taking the odd-numbered cable as an example can get Eq. (7).

$$
\bar{m}_{2 i+1} \ddot{V}_{2 i+1}+\bar{k}_{2 i+1} V_{2 i+1}+\bar{c}_{2 i+1} \dot{V}_{2 i+1}=\bar{f}_{2 i}
$$

where

$$
\begin{gathered}
\bar{m}_{2 i+1}=\int_{0}^{l} \mathbf{N}_{c}^{\mathrm{T}} m_{c} \mathbf{N}_{c} \mathrm{~d} x_{2 i+1} \\
\bar{c}_{2 i+1}=\int_{0}^{l} \mathbf{N}_{c}^{\mathrm{T}} c \mathbf{N}_{c} \mathrm{~d} x_{2 i+1} \\
\bar{k}_{2 i+1}=-\int_{0}^{l} \mathbf{N}_{c}^{\mathrm{T}} T_{2 i+1} \mathbf{N}_{c}^{\prime \prime} \mathrm{d} x_{2 i+1} \\
\bar{f}_{2 i+1}=\int_{0}^{l} \mathbf{N}_{c}^{\mathrm{T}}\left[m_{c}\left(\begin{array}{c}
\frac{x_{2 i+1}}{l} \ddot{w}_{t, 2 i+1} \cos \theta \\
\left.-\frac{x_{2 i+1}}{l} \ddot{v}_{t, 2 i+1} \sin \theta\right)
\end{array}\right]+c\left(\begin{array}{l}
\frac{x_{2 i+1}}{l} \dot{w}_{t, 2 i+1} \cos \theta \\
\left.-\frac{x_{2 i+1}}{l} \dot{v}_{t, 2 i+1} \sin \theta\right)+\Delta T_{2 i+1} \mathbf{N}_{c}^{\prime \prime} \mathbf{V}_{2 i+1}+f_{v, 2 i+1}
\end{array}\right] d x_{2 i+1}\right.
\end{gathered}
$$

Similarly, the other three sets of dynamic equation are also simplified by the Galerkin method.

Even-numbered cable along the $y_{2 i}$ direction:

$$
\begin{gathered}
\bar{m}_{2 i}=\int_{0}^{l} \mathrm{~N}_{c}^{\mathrm{T}} m_{c} \mathbf{N}_{c} \mathrm{~d} x_{2 i} \\
\bar{c}_{2 i}=\int_{0}^{l} \mathbf{N}_{c}^{\mathrm{T}} c \mathbf{N}_{c} \mathrm{~d} x_{2 i} \\
\bar{k}_{2 i}=-\int_{0}^{l} \mathbf{N}_{c}^{\mathrm{T}} T_{2 i} \mathbf{N}_{c}^{\prime \prime} \mathrm{d} x_{2 i}
\end{gathered}
$$




$$
\bar{f}_{2 i}=\int_{0}^{l} \mathbf{N}_{c}^{\mathrm{T}}\left[m_{c}\left(\begin{array}{l}
\frac{x_{2 i}}{l} \ddot{w}_{t, 2 i} \cos \theta \\
\frac{x_{2 i}}{l} \ddot{v}_{t, 2 t} \sin \theta
\end{array}\right)+c\left(\begin{array}{l}
\frac{x_{2 i}}{l} \cos \theta \dot{w}_{t, 2 i} \\
\frac{x_{2 i}}{l} \sin \theta \dot{v}_{t, 2 i}
\end{array}\right)+\Delta T_{2 i} \mathbf{N}_{c}^{\prime \prime} \mathbf{V}_{2 i}+f_{v, 2 i}\right] \mathrm{d} x_{2 i}
$$

Tube along the $y_{t}$ direction:

$$
\begin{gathered}
\bar{m}_{t y}=\int_{0}^{L} \mathbf{N}_{t}^{\mathrm{T}} m_{t} \mathbf{N}_{t} \mathrm{~d} x_{t} \\
\bar{c}_{t y}=\int_{0}^{L} \mathbf{N}_{t}^{\mathrm{T}} c_{t} \mathbf{N}_{t} \mathrm{~d} x_{t} \\
\bar{k}_{t y}=\int_{0}^{L} \mathbf{N}_{t}^{\mathrm{T}} E_{t} I_{t} \mathbf{N}_{t}^{(4)} \mathrm{d} x_{t} \\
\bar{f}_{t y}=-2 \sum_{i=1}^{n} \Delta T_{i} \cos \theta \mathbf{N}_{t i}^{\mathrm{T}}-\int_{0}^{L} \mathbf{N}_{t}^{\mathrm{T}} f_{t y} d x_{t}
\end{gathered}
$$

Tube along the $z_{t}$ direction:

$$
\begin{gathered}
\bar{m}_{t z}=\int_{0}^{L} \mathbf{N}_{t}^{\mathrm{T}} m_{t} \mathbf{N}_{t} \mathrm{~d} x_{t} \\
\bar{c}_{t z}=\int_{0}^{L} \mathbf{N}_{t}^{\mathrm{T}} c_{t} \mathbf{N}_{t} \mathrm{~d} x_{t} \\
\bar{k}_{t z}=\int_{0}^{L} \mathbf{N}_{t}^{\mathrm{T}} E_{t} I_{t} \mathbf{N}_{t}^{(4)} \mathrm{d} x_{t} \\
\bar{f}_{t z}=-2 \sum_{i=1}^{n} \Delta T_{i} \sin \theta \mathbf{N}_{t i}^{\mathrm{T}}-\int_{0}^{L} \mathbf{N}_{t}^{\mathrm{T}} f_{t z} \mathrm{~d} x_{t}
\end{gathered}
$$

Through the above analysis of the simplified model, the coupled dynamic equations of submerged floating tunnel structure system are established. The solution process of dynamic equation adopt the CMS method [32]. The tunnel is regarded as a substructure and each cable is regarded as a substructure, too. Each substructure is 
coupled through the displacement coordination and force balance conditions at the tunnel-cable connection point. As is shown in Fig. 2, the tunnel and the cable are calculated separately. The position state (displacement, velocity, acceleration) of the upper end of the cable at each time step defines the cable boundary conditions. The dynamic response of the cable can be solved under this boundary condition. Then, calculating the cable force, which is used as the external force of the tunnel to solve the dynamic response of the tunnel in next time step. In numerical analysis, adopting a smaller time step can satisfy accuracy requirements. The advantages of this solution method are: (1) each substructure can adopt models with different precision depending on requirement; (2) the analysis of each substructure at each time step can be calculated in parallel, which can greatly improve the calculation efficiency for large-scale problems; (3) asynchronous length can be used to analyze the substructure so that efficiency can be optimized.

\section{Verification of Analytical Modal}

\subsection{Finite Element Model}

In order to verify the accuracy of the simplified model in this article. a finite element model of the floating tunnel is established in this section. So far, no submerged floating tunnel has been built in the world. Based on the conceptual model of Qiandao Lake floating tunnel, this paper selects the parameters in the literature $[14,18,27]$ for model analysis. The specific parameters are shown in Table 1.

Setting two pairs of cables at $L / 3$ and $2 L / 3$ position respectively. Two ends of the tunnel are connected to the foundation by spherical hinges and the cables are fixed at the bottom. Figure 3 is a model of submerged floating tunnel with specific parameters. Obviously, the entire structure is a positive symmetric structure. The model proposed in this paper is adopted to calculate the mid-span displacement of

Fig. 2 CMS method solution process

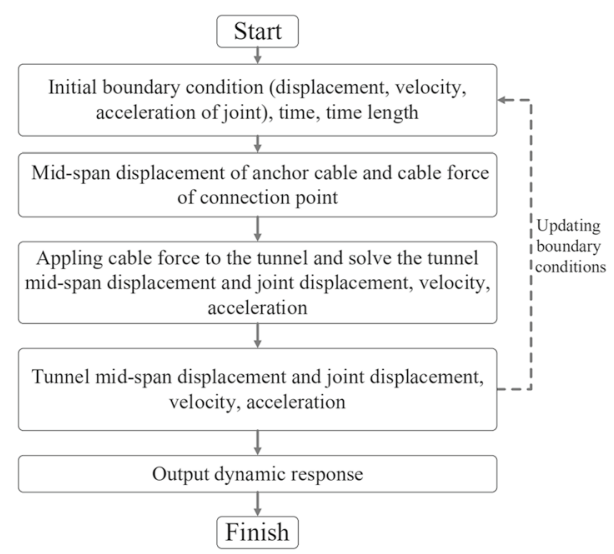


Table 1 Basic parameters of the submerged floating tunnel

\begin{tabular}{llll}
\hline Components & Basic Parameter & Symbol & Symbol \\
\hline No.i Cable & Length & $l$ & $180 \mathrm{~m}$ \\
& Diameter & $d$ & $0.328 \mathrm{~m}$ \\
& Elastic modulus & $E$ & $2.1 \times 10^{11} \mathrm{~Pa}$ \\
& Initial tension & $T_{0}$ & $3.79 \times 10^{7} \mathrm{~N}$ \\
& Inclined mooring angle & $\theta$ & $\pi / 3$ \\
Tunnel & Rayleigh Damping & $\alpha, \beta$ & 5,0 \\
& Length & $L$ & $200 \mathrm{~m}$ \\
& Diameter & $D$ & $15 \mathrm{~m}$ \\
& Elastic modulus & $E_{t}$ & $3.0 \times 10^{10} \mathrm{~Pa}$ \\
& Initial tension & $T_{0}$ & $3.79 \times 10^{7} \mathrm{~N}$ \\
& Flexural rigidity & $I_{t}$ & $2848 \mathrm{~m}^{4}$ \\
\multirow{5}{*}{ Environment } & Density & $\alpha, \beta$ & 5,0 \\
\hline
\end{tabular}
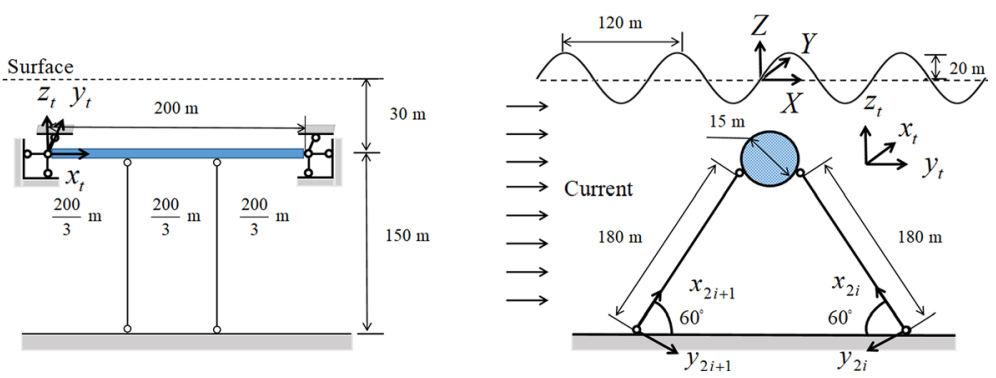

Fig. 3 Parameters of submerged floating tunnel

the tunnel (MSDT), mid-span displacement of the cable (MSDC) and the stress of the cable, which are used as reference indicators.

\subsection{Static Situation}

When the environment effects are not considered, the structural amplitude will decay freely from the initial position. Finally, the entire system will reach equilibrium position. Using the FEA method to analyze the structural equilibrium position, the tunnel adopts BEAM element and the cable adopts LINK element. The initial tension of the cable in Table 1 is equivalent to the initial stress of $448.79 \mathrm{MPa}$ applied to the LINK element in the finite element model. In structural mechanics, the submerged floating tunnel can be regarded as a four-time statically indeterminate 1 structure, The tunnel is equivalent to a beam only considering bending deformation, Because of greatly initial force, none of cable suffers from slack and sudden changes in cable force. Therefore, cables are regarded as truss bar by considering axial deformation only. The force method is used to solve the structure. Case 1: No 
external load applied on the structure; Case 2: Applying a static force of $10^{7} \mathrm{~N}$ at mid-span point of tunnel in the vertical direction; Case 3: Applying a static force of $10^{7} \mathrm{~N}$ at mid-span point of tunnel in the cross direction. The results calculated by the present model, the FEA method and structural mechanics are shown in Table 2.

Static response of structure is shown in Table 2, the mid-span displacement calculated of tube by the present model is $-0.165 \mathrm{~m}$ under the function of initial tension. Compared with the FEA method and the structural mechanics, the deviation is $0.03 \mathrm{~m}$. The cable force of upper end is $295.21 \mathrm{MPa}$ by the present model, compared to the FEA method and the structural mechanics calculation structure, the deviation is less than $7 \mathrm{MPa}$.

In general, the calculation results of the present model are consistent with the results of FEA method and structure mechanics. What's more, the simplified model can maintain a higher calculation efficiency, which shows that the present model has high accuracy in the simulation of the stiffness of original structure.

\subsection{Dynamic Situation}

When the effects of the environment are not considered, the dynamic verification of the structure is carried out. Applying a cross concentrated simple harmonic load $F=10^{7} \sin (\pi t) \mathrm{N}$ at the mid-span position of the tunnel and result is shown in Fig. 4. Then, applying a vertical concentrated simple harmonic load $F=10^{7} \sin (\pi t)$ $\mathrm{N}$ at the mid-span position of the tunnel and result is shown in Fig. 5. Next, Using the FEA method to establish the same model with the same load. Finally, placing the correspondingly dynamic response in Figs. 4 and 5 to make a comparison. For the effect of damping, the vibration of the structure system caused by the initial tension will gradually attenuate. Finally, the structure will vibrate in a steady state under the function of harmonic load.

In the steady-state vibration process of the structure under external loads, the dynamic response of the cable midpoint and tunnel mid span calculated by the present model in Figs. 4 and 5 indicate that dynamic characteristics such as the amplitude, period and phase being similar to the results of the FEA method. The comparison shows that the present model has relatively high accuracy for the rigidity, mass and damping of the original structure.

Table 2 Static response of the submerged floating tunnel at equilibrium position

\begin{tabular}{lllll}
\hline Case & Reference indicators & Present model & FEA method & Structure mechanics \\
\hline 1 & MSDT & $-0.165 \mathrm{~m}$ & $-0.168 \mathrm{~m}$ & $-0.168 \mathrm{~m}$ \\
& cable force & $295.21 \mathrm{MPa}$ & $301.27 \mathrm{MPa}$ & $301.04 \mathrm{MPa}$ \\
2 & MSDT & $-0.180 \mathrm{~m}$ & $-0.183 \mathrm{~m}$ & $-0.183 \mathrm{~m}$ \\
& cable force & $282.52 \mathrm{MPa}$ & $288.34 \mathrm{MPa}$ & $288.13 \mathrm{MPa}$ \\
3 & MSDT & $0.019 \mathrm{~m}$ & $0.019 \mathrm{~m}$ & $0.019 \mathrm{~m}$ \\
& odd-numbered cable force & $304.70 \mathrm{MPa}$ & $310.82 \mathrm{MPa}$ & $310.59 \mathrm{MPa}$ \\
\hline
\end{tabular}




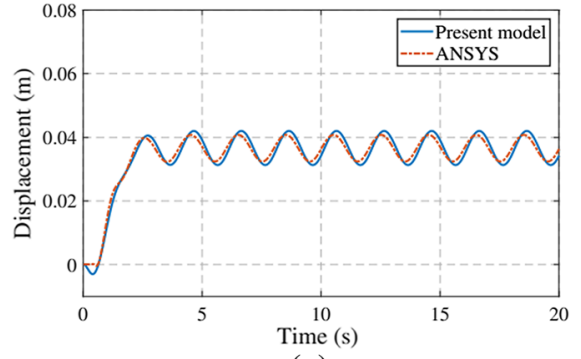

(a)

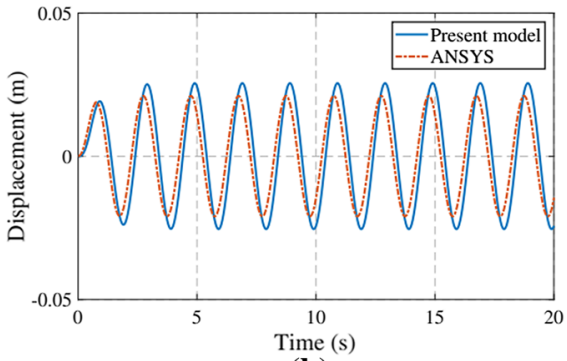

(b)

Fig. 4 Dynamic response of the submerged floating tunnel under the action of $F=10^{7} \sin (\pi t) \mathrm{N}$ at the midpoint of the tunnel in cross direction. a Cross displacement of cable midpoint; $\mathbf{b}$ cross displacement of tunnel midpoint

\section{Case Study}

\subsection{Static Water}

From the initial position, the structure system undergoes free decay vibration and reaches the equilibrium position finally. Through the analysis of Fig. $6 a$ and b, the midpoint of cable produces a cross displacement of $0.0379 \mathrm{~m}$ by considering boundary condition. While the boundary coordination are not considered, the cross displacement of the cable is always zero. So the boundary coordination has a significant impact on the displacement of the cable. Then, through the Fig. $6 \mathrm{c}$ and d, it can be found that the displacement of the tunnel midpoint is $0.1649 \mathrm{~m}$ in two cases. Finally, comparing the Fig. 6e and f diagrams, the cable axial stress is $295.20 \mathrm{MPa}$, which indicates the existence of boundary coordination has basically no effect on the calculation of tunnel motion and cable force.

The dynamic force of the cable is caused by the displacement of upper end position and deformation of cable. The result shows that cross displacement of cable

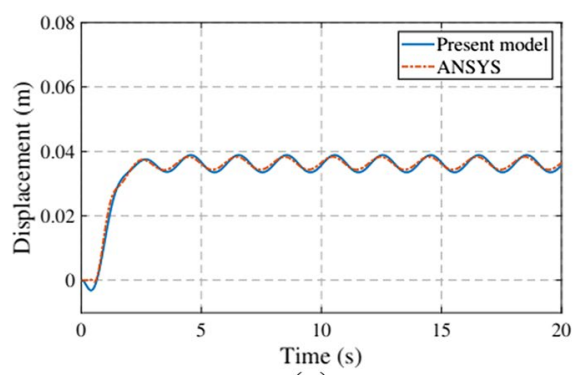

(a)

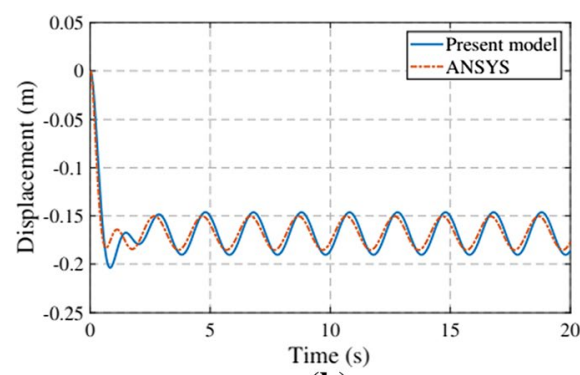

(b)

Fig. 5 Dynamic response of the submerged floating tunnel under the action of $F=10^{7} \sin (\pi t) \mathrm{N}$ at the midpoint of the tunnel in vertical direction. a Cross displacement of cable midpoint; $\mathbf{b}$ vertical displacement of tunnel midpoint 
point is $0.0379 \mathrm{~m}$, which is a relatively small amount compared to cable length with $180 \mathrm{~m}$. Therefore, the elongation of the cable caused by bending deformation is much smaller than the elongation of the upper end position. The introduction of boundary conditions can use approximate methods to accurately calculate the deformation of the cable, but the elongation caused by the deformation of the cable is relatively small. When the research object is the tunnel, the existence of boundary conditions has little effect. The response of the structure is very small compared to the size of the structure from the results. So it is reasonable to complete the relevant theoretical derivation based on the premise of small deformation in the above chapter.



(a)

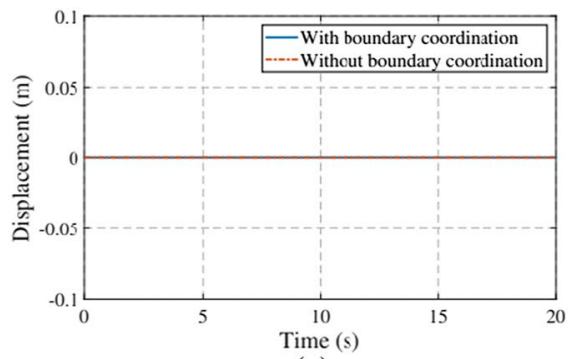

(c)

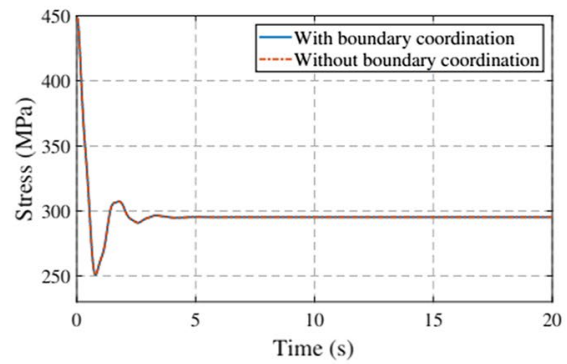

(e)

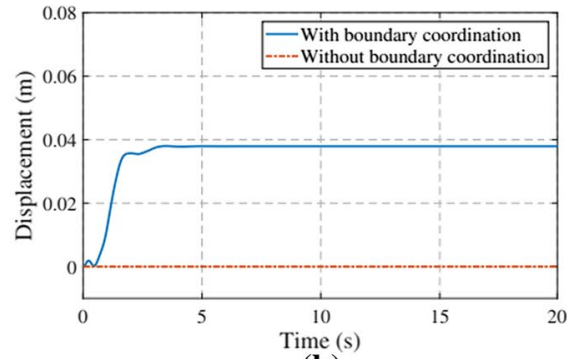

(b)

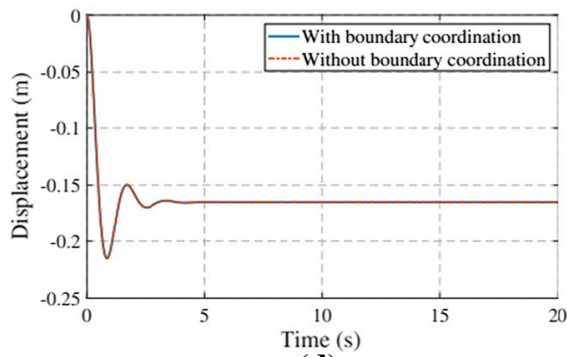

(d)



(f)

Fig. 6 Response of the submerged floating tunnel in static water. a Cross displacement of odd-numbered cable midpoint; b cross displacement of even-numbered cable midpoint; $\mathbf{c}$ cross displacement of tube midpoint; $\mathbf{d}$ vertical displacement of tube midpoint; $\mathbf{e}$ stress of odd-numbered cable at upper end; $\mathbf{f}$ stress of even-numbered cable at upper end 


\subsection{Current Flow}

The maximum current velocity is $0.6 \mathrm{~m} / \mathrm{s}$ in the Taiwan Channel and $1.7 \mathrm{~m} / \mathrm{s}$ in Bohai Channel. The environment of the Qiongzhou Channel is the worst and the maximum current velocity can reach $3.06 \mathrm{~m} / \mathrm{s}$. In this section, the steady flow of $0-5 \mathrm{~m} / \mathrm{s}$ are used to study the influence of the current velocity to submerged floating tunnel.

It can be seen from Fig. 7a and $\mathrm{b}$ that the current velocity has an obvious influence to the midpoint of the cable. As the current velocity increases, the midpoint displacement of the odd-numbered cable becomes larger. Because the current causes the tube to produce horizontal displacement and increased the elongation of the cable along the string's direction. while the midpoint displacement of the even-numbered cable decreases with the increase of current velocity when current velocity is small. Then, the displacement increases with the increment of current velocity. Because when the current flow is relatively small, the initial tension of the cable takes a dominant position in the displacement so the current flow has a weakening effect than the initial tension. With the raise of the current velocity, current gradually takes up the dominant position. Comparing Fig. 7c and d, it can be found that under the action of current, the tunnel will produce relatively small cross displacement, but when the current velocity is greater than $3 \mathrm{~m} / \mathrm{s}$, the influence is significantly increased. while the vertical displacement of the tube by the current is not influenced, because the current in the perpendicular direction of the cable and the current flow in the horizontal direction of the tube are considered in the calculation. In fact, this effect is equivalent to the anti-symmetric load in structural mechanics so that the vertical displacement of the tunnel has no effect. For odd-numbered cable, the stress gradually increases under the action of current, which has a "loading" effect. This is due to the horizontal displacement of the tunnel caused by the current, which increases dynamic displacement of the upper end of the cable along the string's direction. For the even-numbered cable, the horizontal displacement of the tunnel caused by the current has an "unloading" effect.

\subsection{Wave Action}

Wave action is the main load of submerged floating tunnel, which affects the safety and stability of the submerged floating tunnel. Therefore, studying the effect of waves on submerged floating tunnel is one of the issues that must be considered in the design and operation period.

This section uses the hydrological environment of three channels as the background to design parameters. As is shown in Table 3. Medium and small amplitude wave theory is adopted to describe wave motion.

Using the present model in this paper to solve the dynamic response of submerged floating tunnel. Case 1 considers the cross and vertical velocity and acceleration of the wave; Case 2 only considers the cross velocity and acceleration components of the wave; Case 3 only considers the vertical velocity and acceleration components of the wave. The result is shown in Fig. 8. 


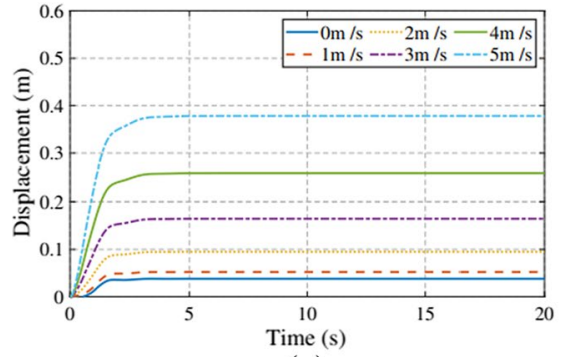

(a)

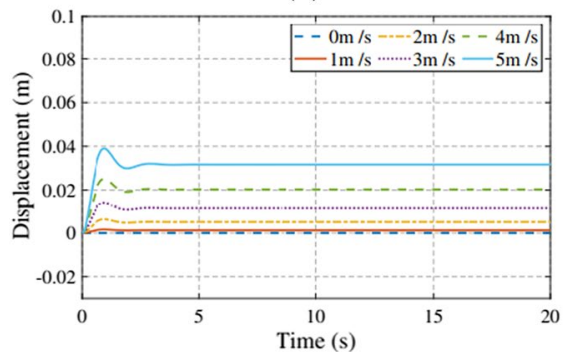

(c)

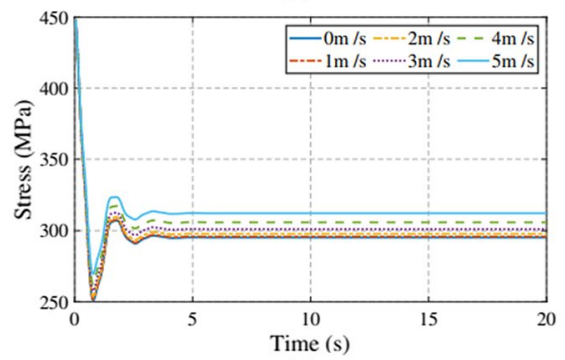

(e)

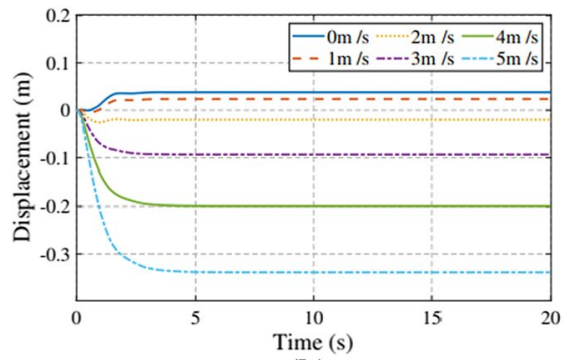

(b)



(d)

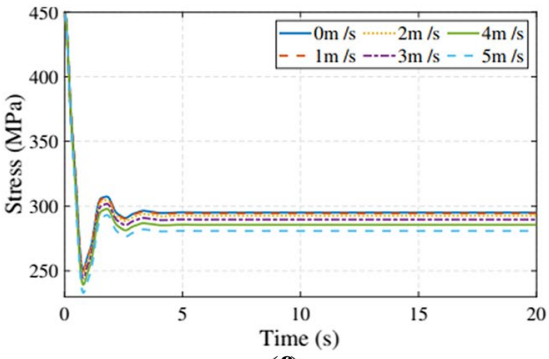

(f)

Fig. 7 Dynamic response of the submerged floating tunnel under Steady flow. a Cross displacement of odd-numbered cable midpoint; $\mathbf{b}$ cross displacement of even-numbered cable midpoint; $\mathbf{c}$ cross displacement of tunnel midpoint; $\mathbf{d}$ vertical displacement of tunnel midpoint; e stress of odd-numbered cable at upper end; $\mathbf{f}$ stress of even-numbered cable at upper end

It can be seen from Fig. 8a and $b$ that the amplitude of the odd-numbered cable and the even-numbered cable midpoint is $2.05 \mathrm{~cm}$ under the action of the waves, $1.85 \mathrm{~cm}$ under the action of the cross component of the waves and $0.80 \mathrm{~cm}$ under the action of the cross component of the waves, which indicates that the cross component of the wave has a greater effect on the amplitude of the cable midpoint. When paying attention to the dynamic displacement response of the of cable midpoint under the action of the wave, the cross component of the wave should be considered.

From Fig. $8 \mathrm{c}$ and d, the cross and vertical response of the tube midpoint under the action of wave can be completely overlapped to cross response only under the action of the cross component and the vertical response only under the action of the 
Table 3 Basic parameters of waves

\begin{tabular}{llllll}
\hline Basic parameters & Hight & Length & Period & Wave number & Depth \\
\hline Symbol & $H w(\mathrm{~m})$ & $L w(\mathrm{~m})$ & $T w(\mathrm{~s})$ & $\kappa(/ \mathrm{m})$ & $d w(\mathrm{~m})$ \\
Value & 20 & 120 & 10.8 & 0.0345 & 180 \\
\hline
\end{tabular}

vertical component of wave, which indicates that the dynamic response of the structure exhibits a linear relationship under the action of wave.

As is shown in Fig. 8e and $\mathrm{f}$, the amplitude of the odd-numbered cable force is 40.1 MPa under the action of the wave, 26.2 MPa under the action of the cross component of the waves and $34.8 \mathrm{MPa}$ under the action of the vertical component of

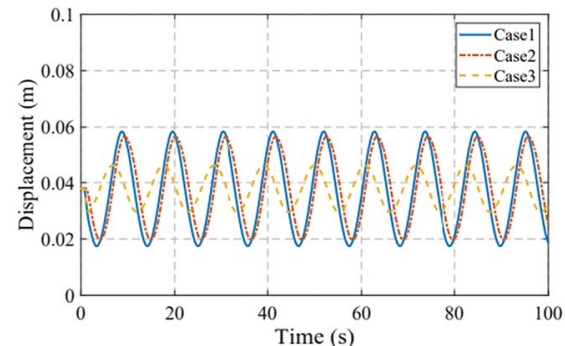

(a)

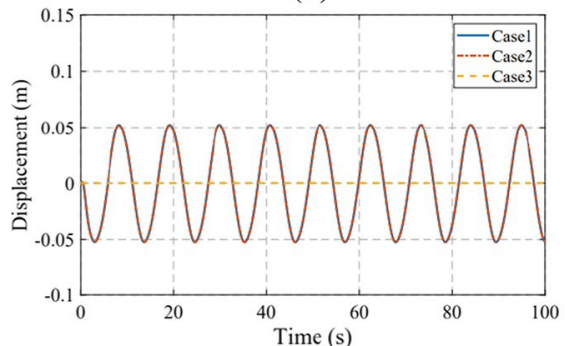

(c)

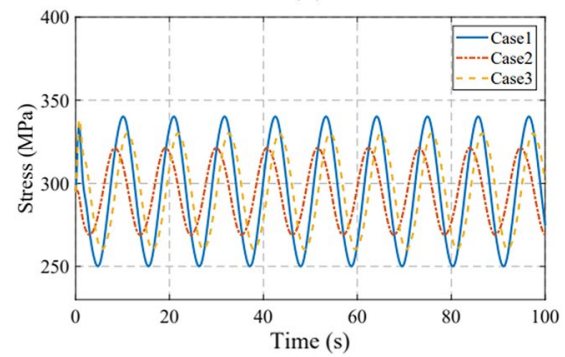

(e)

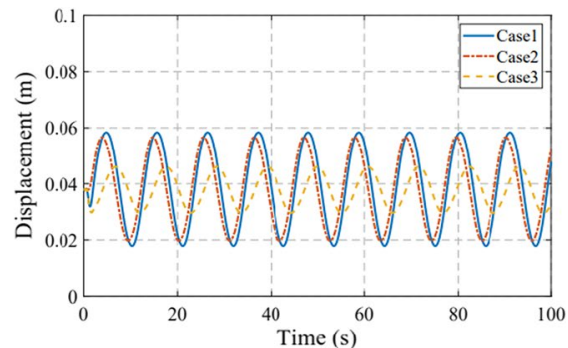

(b)

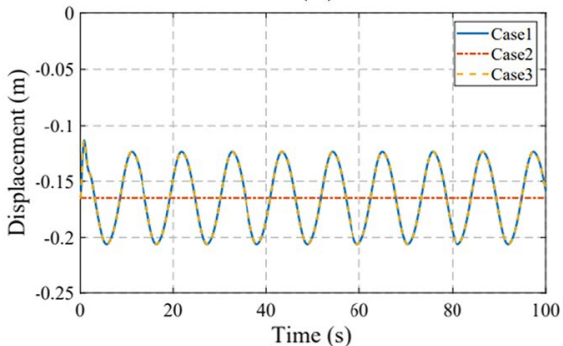

(d)

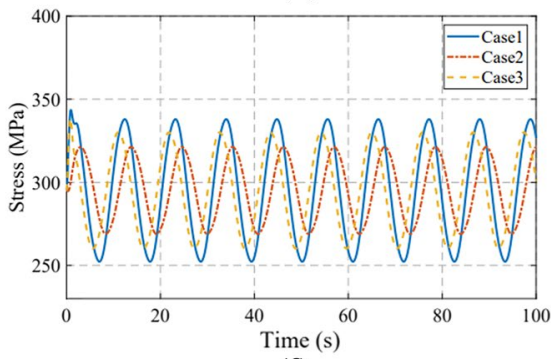

(f)

Fig. 8 Dynamic response of the submerged floating tunnel under wave action. a Cross displacement of odd-numbered cable midpoint; $\mathbf{b}$ cross displacement of even-numbered cable midpoint; $\mathbf{c}$ cross displacement of tunnel midpoint; $\mathbf{d}$ vertical displacement of tunnel midpoint; e stress of odd-numbered cable at upper end; $\mathbf{f}$ stress of even-numbered cable at upper end 
the waves, which indicates that the vertical component of the wave has a greater effect on the amplitude of the cable midpoint. When paying attention to the dynamic response of the cable force under the action of the waves, the vertical component of the wave should be considered.

\section{Conclusion}

This paper proposes a dynamic model of submerged floating tunnel for the coupled vibration in space. The tube can vibrate along the cross and vertical directions, which can solve the problem of analyzing the horizontal current and vertical wave component of submerged floating tunnel. Compared with the method of using mode superposition to calculate the cable response, this paper solves the problem of the incoordination between the tunnel and the cable connection by introducing the shape function so that the model can satisfy the force balance and boundary coordination conditions at the same time, which improves the calculation accuracy. This paper adopts the CMS method, which makes the calculation more applicable and meets the needs of large-scale and long-span engineering structure. And through case analysis, the following conclusions can be drawn.

1. The introduction of boundary conditions has little effect on the dynamic response of the tube, but it has a significant impact on the dynamic response of the cable. Therefore, when concentrating the vibration of the cable, the boundary conditions should be considered.

2. The cross water flow has minimal effect on the static equilibrium position of the tunnel, but it has a significant effect on the displacement of the cable midpoint.

3. The cross and vertical components of the wave present a linear relationship with the action of the cable. The displacement of the cable midpoint is mainly determined by the cross components of the water wave, while the upper end cable force of the cable is mainly affected by the vertical components of wave.

Author contributions "LC carried out the studies, participated in the derivation and drafted the manuscript. ZM carried out the numerical solution. LL participated in the FEA. CY participated in the design of the study, HF conceived of the study, and participated in its design and coordination and helped to draft the manuscript. ZJ performed the case study. All authors read and approved the final manuscript."

Funding This work is supported by the National Nature Science Foundation of China (Grant No.12002279); the Fundamental Research Funds for the Central Universities, NWPU (G2020KY05307).

Availability of data and material We do not have any data or material that cannot be shared openly.

\section{Declarations}

Conflict of interests The authors declare that they have no known competing financial interests or personal relationships that could have appeared to influence the work reported in this paper. The authors declare that they have no competing interests. 
Ethics approval and consent to participate The authors approve and consent to participate.

Consent for publication The Author agrees to publication.

Open Access This article is licensed under a Creative Commons Attribution 4.0 International License, which permits use, sharing, adaptation, distribution and reproduction in any medium or format, as long as you give appropriate credit to the original author(s) and the source, provide a link to the Creative Commons licence, and indicate if changes were made. The images or other third party material in this article are included in the article's Creative Commons licence, unless indicated otherwise in a credit line to the material. If material is not included in the article's Creative Commons licence and your intended use is not permitted by statutory regulation or exceeds the permitted use, you will need to obtain permission directly from the copyright holder. To view a copy of this licence, visit http://creativecommons.org/licen ses/by/4.0/.

\section{References}

1. Larssen, R.M., Jakobsen, S.E.: Submerged floating tunnels for crossing of wide and deep fjords. Procedia Eng. 4, 171-178 (2010)

2. Minoretti, A., Xiang, X., Johansen, I.L., Eidem, M.: The future of the tunnel crossing: the submerged floating tube bridge. Struct. Eng. Int. 30(4), 1-5 (2008)

3. Andrew, C.E.: Floating tunnel for longwater crossings. Trans. Am. Soc. Civ. Eng. 116(12), 708-720 (1951)

4. Ingerslev, C.L.: Innovations in resilient infrastructure design: immersed and floating tunnels. Civ. Eng. 165(6), 52-58 (2012)

5. Xiang, Y.Y., Yang, Y.: Challenges and technical ideas in the construction of China's coastal crossstrait passage. China Munic. Eng. 5, 1-5 (2016)

6. Gang, Y.: Three-dimensional analysis and segmental model experimental investigation of submerged floating tunnel. In: Zhejiang University. Hangzhou, China (2003)

7. Sun, S.N.: Dynamic response analysis of submerged floating tunnel. In: Dalian University of Technology, Dalian, China (2008)

8. Mai, J.T.: The study on responses of a submerged floating tunnel subjected to the wave and current. In: Southwest Jiaotong University. Chengdu, China (2005)

9. Wang, G.D.: Numerical analysis and experiment research of submerged floating tunnel subjected to wave and current effect. In: Southwest Jiaotong University. Chengdu, China (2005)

10. Xiang, Y.Y., Liu, C., Zhang, K., Wu, Q.: Risk analysis and management of submerged floating tunnel and its application-ScienceDirect. Procedia Eng. 4(1), 107-116 (2010)

11. Joachim, E., Adrian, C.: Nonlinear water waves with applications to wave-current interactions and tsunamis. Jahresber. Deutschen Mathematiker-Vereinigung 114, 3 (2012)

12. Zhang, H.P., Lu, K., Zhang, W.: Investigation on dynamic behaviors of rotor system with looseness and nonlinear supporting. Mech. Syst. Signal Process. 166(10), 108400 (2022)

13. Lu, W., Ge, F., Wang, L.: On the slack phenomena and snap force in tethers of submerged floating tunnels under wave conditions. Mar. Struct. 24(4), 358-376 (2011)

14. Lin, H., Xiang, Y., Yang, Y.: Dynamic response analysis for submerged floating tunnel due to fluidvehicle-tunnel interaction. Ocean Eng. 166, 290-301 (2018)

15. Xiang, Y., Yang, Y.: Spatial dynamic response of submerged floating tunnel under impact load. Mar. Struct. 53, 20-31 (2016)

16. Xiang, Y., Chen, Z., Yang, Y., Lin, H., Zhu, S.: Dynamic response analysis for submerged floating tunnel with anchor-cables subjected to sudden cable breakage. Mar. Struct. 59, 179-191 (2018)

17. Xiang, Y., Chao, C.: Vortex-induced dynamic response analysis for the submerged floating tunnel system under the effect of currents. J. Waterway Port Coast. Ocean Eng. 139(3), 183-189 (2013)

18. Chen, Z., Xiang, Y., Lin, H., Yang, Y.: Coupled Vibration Analysis of Submerged Floating Tunnel System in Wave and Current. Appl. Sci. 8, 8 (2018)

19. Lu, K., Jin, Y.L., et al.: The applications of POD method in dual rotor-bearing systems with coupling misalignment. Mech. Syst. Signal Process. 150, 107236 (2021) 
20. DiPilato, M., Perotti, F., Fogazzi, P.: 3D dynamic response of submerged floating tunnels under seismic and hydrodynamic excitation. Eng. Struct. 30(1), 268-281 (2008)

21. Long, X., Ge, F., Wang, L.: Effects of fundamental structure parameters on dynamic responses of submerged floating tunnel under hydrodynamic loads. Acta. Mech. Sin. 3, 335-344 (2009)

22. Martinelli, L., Barbella, G., Feriani, A.: A numerical procedure for simulating the multi-support seismic response of submerged floating tunnels anchored by cables. Eng. Struct. 33(10), 2850-2860 (2011)

23. Cristian, C., Kim, S., Kim, M.H., Park, W.S.: Numerical simulation of the coupled dynamic response of asubmerged floating tunnel with mooring lines in regular waves. Ocean Syst. Eng. 5(2), 109-123 (2008)

24. Jin, C.; Kim, M.H.; Choi, J.: Coupled dynamics simulation of submerged floating tunnel for various system parameters and wave conditions. In: ASME 2018 37th international conference on ocean, offshore and arctic engineering (2018)

25. Chen, J.Y., Li, J., Sun, S.N., Su, Z.B.: Experimental and numerical analysis of submerged floating tunnel. J. Central South Univ. 19(10), 2949-2957 (2012)

26. Chao, C.F.: Dynamic response analysis and experiment of submerged floating tunnel based on fluidstructure interaction. In: Zhejiang University. Hangzhou, China (2020)

27. Seo, S.I., Mun, H.S., Lee, J.H., Kim, J.H.: Simplified analysis for estimation of the behavior of a submerged floating tunnel in waves and experimental verification. Mar. Struct. 44, 142-158 (2015)

28. Clough, R.W., Penzien, J., Griffin, D.S.: Dynamics of Structures, 2nd edn. McGraw-Hill, New York (1993)

29. Paik, I.Y., Oh, C.K., Kwon, J.S.: Analysis of wave force induced dynamic response of submerged floating tunnel. KSCE J. Civ. Eng. 8(5), 543-550 (2004)

30. Wu, Z., Ni, P., Mei, G.: Vibration response of cable for submerged floating tunnel under simultaneous hydrodynamic force and earthquake excitations. Adv. Struct. Eng. 2018, 136 (2018)

31. Xiang, Y., Zhang, K.: The layered integrating method for calculating wave force of submerged floating tunnel based on Morison equation. J. Zhejiang Univ. Eng. Sci. 45, 1399-1404 (2011)

32. Chen, L., Xu, Y.S.L.: A component mode synthesis method for reduced-order modeling of cable networks in cable-stayed bridges. J. Sound Vibr. 2020, 115769 (2020) 